\title{
CARACTERIZACIÓN POR TÉCNICAS ESPECTROSCÓPICAS DEL O-CARBOXIMETILQUITOSANO OBTENIDO POR DERIVATIZACIÓN DEL QUITOSANO
}

\author{
Susana Cusihuamán Noa ${ }^{\mathrm{a}}$, María Elena Talavera Núñez ${ }^{\mathrm{b}}$, Carlos Arenas Chávez \\ David G. Pacheco Salazar ${ }^{\mathrm{d}}$, Corina Vera Gonzales ${ }^{\mathrm{b}}$
}

\begin{abstract}
RESUMEN
El O-carboximetilquitosano (O-CMQ) fue derivatizado a partir del copolímero quitosano por el método de alquilación directa. Este método implicó la reacción del quitosano con ácido monocloroacético previa alcalinización. El producto derivatizado mostró solubilidad en un rango de $\mathrm{pH}$ de $1-13$ y a $\mathrm{pH}=5$ exhibió baja solubilidad. El grado de sustitución (GS) fue de $0,92 \mathrm{~mol} / \mathrm{mol}$, determinado por titulación conductimétrica. Los resultados de Espectroscopia Infrarroja con Transformada de Fourier (FT-IR) permitieron identificar una banda de absorción de intensidad fuerte a $1585 \mathrm{~cm}^{-1}$, correspondiente al estiramiento del $\mathrm{C}=\mathrm{O}$, lo que indica la presencia de un anión carboxílico $\left(-\mathrm{COO}^{-}\right)$, que es característico del O-carboximetilquitosano. Mediante Espectroscopia de Resonancia Magnética Nuclear de Hidrógeno y del carbono $\left({ }^{1} \mathrm{H} \mathrm{NMR},{ }^{13} \mathrm{C} \mathrm{NMR}\right)$ se mostró que la sustitución se llevó a cabo en el carbono 3 y 6 del quitosano. El O-CMQ a concentración de $50 \mathrm{mg} / \mathrm{mL}$, incrementó la supervivencia celular en un $8 \%$ frente a la toxicidad del $\mathrm{Pb}$ (II) a una concentración de $41,44 \mathrm{mg} / \mathrm{mL}$.
\end{abstract}

Palabras clave: Quitosano, O-carboximetilquitosano, grado de sustitución, solubilidad.

\section{CHARACTERIZATION BY SPECTROSCOPIC TECHNIQUES OF O-CARBOXIMETILQUITOSANO OBTAINED BY DERIVATIZATION OF THE CHITOSAN}

\begin{abstract}
The O-carboxymethylchitosan (O-CMQ) was derivatized from the chitosan copolymer by the direct alkylation method. This method involved the reaction of the chitosan with monochloroacetic acid after alkalinization. The derivatized product showed solubility

\footnotetext{
${ }^{a}$ Escuela Profesional de Química, Universidad Nacional de San Agustín de Arequipa.

b*Departamento Académico de Química, Universidad Nacional de San Agustín de Arequipa. Av. Independencia s/n, Arequipa, Perú. Email: cverag@unsa.edu.pe.

c Departamento Académico de Biología, Universidad Nacional de San Agustín de Arequipa.

¿ Departamento Académico de Física, Universidad Nacional de San Agustín de Arequipa.
} 
in a $\mathrm{pH}$ range of $1-13$ and low solubility at $\mathrm{pH}=5$. The degree of substitution (GS) was 0,92 , determined by conductometric titration. The results of Fourier Transform Infrared Spectroscopy (FT-IR) allowed us to identify a strong intensity absorption band at $1585 \mathrm{~cm}^{-1}$, corresponding to the stretching of $\mathrm{C}=\mathrm{O}$, which indicates the presence of a carboxylic anion $\left(-\mathrm{COO}^{-}\right)$that is characteristic of O-carboxymethylchitosan (O-CMQ). Using the Nuclear Magnetic Resonance spectroscopy of proton and carbon-13 ( ${ }^{1} \mathrm{H}$ NMR, ${ }^{13} \mathrm{C}$ NMR) showed that the substitution was carried out on carbon 3 and 6 of chitosan. The derivatized O-CMQ at concentration of $50 \mathrm{mg} / \mathrm{mL}$, increased cell survival by $8 \%$ compared to the toxicity of $\mathrm{Pb}$ (II) at concentration of $41,44 \mathrm{mg} / \mathrm{mL}$.

Key words: Chitosan, O-carboxymethylchitosan, degree of substitution, solubility.

\section{INTRODUCCIÓN}

La quitina es el segundo biopolímero natural más abundante después de la celulosa, su estructura química está constituida por monómeros de 2-acetamido-2desoxi-D-glucopiranosa unidos entre sí por enlaces $\beta(1,4)$. El quitosano es un copolímero derivado desacetilado de la quitina, de 2 amino-2-desoxi-D-glucopiranosa y 2-acetamido-2-desoxi-D-glucopiranosa, encontrándose presente en crustáceos, insectos y hongos. Debido a sus propiedades singulares tales como: no toxicidad, biodegradabilidad y biocompatibilidad; el quitosano tiene una amplia gama de aplicaciones en diversos campos: industria de los alimentos, cosméticos, farmacéuticos, biomédicos, tratamiento de aguas y agricultura ${ }^{1}$.

Muchas de las aplicaciones del quitosano se ven afectadas por su escasa solubilidad a pH neutro o alcalino, debido a su estructura cristalina muy estable derivada de enlaces de hidrógeno muy fuertes. Los grupos amino del quitosano son protonados cuando el $\mathrm{pH}$ es menor a 6,5 por lo que muestra solubilidad en soluciones ácidas.

La limitada solubilidad del quitosano se puede superar por despolimerización parcial y modificaciones químicas de este polímero, generando derivados solubles en agua ${ }^{2}$.

Sun et al. ${ }^{3}$ realizaron estudios de un nuevo material adsorbente a base de quitosano para mejorar la selectividad de la adsorción de metales pesados, sintetizando una resina de iones $\mathrm{Pb}$ (II) adsorbidos en N,O-carboximetilquitosano reticulada con glutaraldehido. Por otro lado, muestran que la capacidad de adsorción depende de la cantidad de agente reticulante, grado de sustitución del carboximetilquitosano, valor del pH de la solución inicial y el tiempo de adsorción. En los experimentos de adsorción demostraron que el CMQ reticulado tiene una alta selectividad de adsorción para los iones $\mathrm{Pb}$ (II) en solución que contiene iones metálicos individuales o la coexistencia de tres metales iones de $\mathrm{Cu}$ (II), $\mathrm{Zn}$ (II) y $\mathrm{Pb}$ (II).

En el presente trabajo se sintetizó el O-carboximetilquitosano y se caracterizó mediante técnicas espectroscópicas como FT-IR, RMN de ${ }^{1} \mathrm{H}$ y ${ }^{13} \mathrm{C}$. Por otro lado, se evaluó el efecto citoprotector del O-CMQ frente iones plomo. 


\section{PARTE EXPERIMENTAL}

\section{Reactivos}

Quitosano (peso molecular medio, grado de desacetilación 84,1 \%, viscosidad 525cps), ácido monocloroacético (reactivo ACS, $\geq 99 \%$ ) obtenidos de Sigma Aldrich (St. Louis, MO). Hidróxido de sodio (p.a. $\geq 99 \%$ ), etanol absoluto (p.a. $\geq 99,8 \%$ ) adquiridos de la empresa Merck Millipore. Agua ultrapura Tipo 2.

\section{Síntesis de carboximetilquitosano}

El O-CMQ fue sintetizado a partir de quitosano usando el método de alquilación directa ${ }^{4}$. Para la síntesis del O-CMQ, fue disuelto el hidróxido de sodio $(6,25 \mathrm{~g})$ en $17 \mathrm{~mL}$ de la mezcla agua-isopropanol (1:1). Luego $1 \mathrm{~g}$ de quitosano fue dispersado dentro de la solución anterior y se mantuvo a $25^{\circ} \mathrm{C}$ por 8 horas bajo agitación constante para hinchar y alcalinizar la red polimérica, conformando esta la solución alcalinizada.

Por otro lado, en un vaso de precipitado fue disuelto $6 \mathrm{~g}$ de ácido monocloroacético en 7,5mL de isopropanol y se agregó gota a gota a la solución alcalinizada con agitación constante a $50^{\circ} \mathrm{C}$ de temperatura, durante 30 minutos. Se continuó el proceso de reacción por 6 horas, al finalizar el tiempo se detuvo la reacción agregando $25 \mathrm{~mL}$ de etanol al $70 \%$; obteniéndose el sólido O-CMQ, el que fue filtrado y lavado con etanol al $90 \%$ para desalar y deshidratar, hasta lograr un $\mathrm{pH}$ neutro en los lavados. Finalmente, el O-CMQ fue secado por 12 horas en una estufa a $40 \pm 5^{\circ} \mathrm{C}$ y conservado en un desecador para las pruebas de caracterización posteriores.

\section{Caracterización}

\section{Solubilidad en agua}

La solubilidad del quitosano (Q) y del O-CMQ en medio acuoso a diferentes $\mathrm{pH}$ fue evaluado mediante la determinación de la turbidez por espectrofotometría UV-visible a una longitud de onda de 450nm, basado en el método descrito por Chen y Park 5 .

Se preparó una solución de quitosano y otra de O-CMQ a una concentración de 1,5g/Ly el $\mathrm{pH}$ fue ajustado utilizando ácido clorhídrico $(0,5 \%)$ e hidróxido de sodio $(0,5 \%)$. Se realizó la lectura en un espectrofotómetro uv-visible Gold-Spectrumlab 54 y cuando la absorbancia fue mayor a 0,070 o menor a $85 \%$ de transmitancia, se consideró insoluble.

\section{Análisis conductimétrico}

El grado de sustitución (GS) es el número relativo de los grupos carboximetilados en la cadena del quitosano, el cual fue determinado usando el método conductimétrico, como lo indica Vaghani et al. ${ }^{7}$. Se preparó una solución disolviendo $0,1 \mathrm{~g}$ de carboximetilquitosano en $\mathrm{HCl}$ 0,05M, posteriormente se ajustó el $\mathrm{pH}$ a 2,0-2,2 con NaOH 0,1 M. La titulación se llevó a cabo a $25 \pm 0,5^{\circ} \mathrm{C}$, agregando $0,5 \mathrm{~mL}$ de $\mathrm{NaOH} 0,1 \mathrm{M}$ para cada medida conductimétrica, hasta llegar a un $\mathrm{pH}$ de 11,5. La curva de valoración se muestra en la figura 1, el GS se calculó usando la siguiente ecuación 1: 


$$
G S=\frac{\left(V_{2}-V_{1}\right)}{\left(V_{3}-V_{2}\right)} \times G D
$$

Donde GS es el grado de sustitución del O-CMQ (mol/mol del total de monómeros); GD es el grado de desacetilación del quitosano original; $\mathrm{V}_{1}$ es el volumen de base añadida para alcanzar el primer punto de inflexión; $\mathrm{V}_{2}$ es el volumen de base añadida para alcanzar el segundo punto de inflexión y $\mathrm{V}_{3}$ es el volumen de base añadida para alcanzar el tercer punto de inflexión ${ }^{7}$.

\section{Peso molecular}

Para obtener el peso molecular del carboximetilquitosano (O-CMQ), se determinó la viscosidad intrínseca del mismo, disolviendo $50 \mathrm{mg}$ del O-CMQ (previamente secada a $45^{\circ} \mathrm{C}$ por $24 \mathrm{~h}$ ) en $25 \mathrm{ml}$ de agua destilada, luego se mantuvo en agitación constante por 24 horas, seguidamente se añadió $25 \mathrm{ml}$ de solución de $\mathrm{NaCl} 0,2 \mathrm{~mol} / \mathrm{L}$ y se continuo la agitación por 30 minutos más. La solución se filtró en membrana con diámetro de los poros de $0,45 \mu \mathrm{m}$ (Millipore - White SCWP).

A partir de esta solución se preparó soluciones a las distintas concentraciones $(0,05 \%, 0,1 \%$, $0,2 \%, 0,4 \%$ y $0,6 \%$ ) en $\mathrm{NaCl} 0,1 \mathrm{~mol} / \mathrm{L}$.

Se tomó una alícuota de $10 \mathrm{~mL}$ de cada una de las soluciones y se registró el tiempo de caída en el viscosímetro de vidrio (Ostwald), las medidas se realizaron en baño maría a $30,0 \pm 0,5^{\circ} \mathrm{C} 8$.

\section{Espectroscopia FT/IR}

El espectro infrarrojo fue obtenido de un Espectrofotómetro Infrarrojo con Transformada de Fourier, marca SHIMADZU affinity1 con accesorio de reflectancia total atenuada (ATR). La muestra seca fue colocada en el cristal ATR directamente. El espectro obtenido fue de 750$4000 \mathrm{~cm}^{-1}$ de longitud de onda con $4 \mathrm{~cm}^{-1}$ de resolución.

\section{Resonancia magnética nuclear}

La espectroscopia de resonancia magnética nuclear de ${ }^{1} \mathrm{H}$ y ${ }^{13} \mathrm{C}$ se empleó para desarrollar la elucidación estructural del copolímero. El espectro de la muestra se obtuvo del espectrómetro Bruker Avance III HD $500 \mathrm{MHz}(11,75$ T) de la Pontificia Universidad Católica del Perú, Departamento de Ciencias - Sección Química.

Previo análisis, la muestra fue secada durante 24 horas. Luego, se disolvió $0,0293 \mathrm{~g}$ de O-CMQ en $0,9 \mathrm{~mL}$ de $\mathrm{D}_{2} \mathrm{O}$ y se añadió $0,1 \mathrm{~mL}$ de TSP $(1,25 \mathrm{mg} / \mathrm{mL})$ para la referencia del espectro de $1 \mathrm{H}-\mathrm{RMN}$.

Evaluación de la actividad biológica del O-CMQ

\section{Cultivo celular}

Los linfocitos humanos, fueron separados a partir de sangre total en un gradiente de densidad de Ficoll (polímero de alto peso molecular que permite la separación de las células 
mononucleares de los eritrocitos) y se cultivaron en medio DMEM (Dulbecco's Modified Eagle's Medium), suplementado con suero fetal bovino $10 \%$, penicilina $(100 \mu \mathrm{g} / \mathrm{mL})$, estreptomicina $(100 \mu \mathrm{g} / \mathrm{mL})$ y finalmente fueron incubadas en una cámara de $\mathrm{CO} 2$ al $5 \%$ a $37^{\circ} \mathrm{C}$.

Los linfocitos se sembraron a una densidad de $1 \times 10^{6}$ células / pocillo en placas de cultivo celular de 24 pocillos y se mantuvieron en condiciones de cultivo estándar. Después de 24 horas se renovó el medio de cultivo y se evaluó el efecto citoprotector.

\section{Efecto citoprotector}

Se evaluó el efecto citoprotector del O-CMQ a una concentración de $0,5 \mathrm{mg} / \mathrm{mL}$, frente a concentraciones de plomo $\left(\mathrm{PbCl}_{2}\right) 41,44$ y $82,88 \mathrm{mg} / \mathrm{mL}$. Como blanco se utilizó leucocitos sin tratamiento. Pasada las 48 horas de incubación se renovó el medio de cultivo y se realizó el ensayo de reducción metabólica del bromuro de 3-(4,5- dimetiltiazol-2-ilo)-2,5difeniltetrazol (MTT).

\section{Ensayo MTT}

Este ensayo se basa en la reducción metabólica del bromuro de 3-(4,5- dimetiltiazol-2-ilo)2,5-difeniltetrazol (MTT) realizada por la enzima mitocondrial succinato-deshidrogenasa, produciéndose un compuesto coloreado de color azul (formazán), permitiendo determinar la funcionabilidad mitocondrial de las células tratadas. Este método ha sido muy utilizado para medir supervivencia y proliferación celular. La cantidad de células vivas es proporcional a la cantidad de formazán producido. Para este ensayo, se agregó a cada tratamiento $100 \mu \mathrm{L}$ de MTT. Después de una hora las células son retiradas de la placa de cultivo y se centrifugó por 5 minutos a $13000 \mathrm{rpm}$. Luego, se disolvió los cristales de formazán en $150 \mu \mathrm{L}$ de KOH $2 \mathrm{M}$ y $100 \mu \mathrm{L}$ de dimetil sulfóxido (DMSO), se aforó a $500 \mu \mathrm{L}$ con agua destilada y se midió la absorbancia a $570 \mathrm{~nm}^{9}$.

El porcentaje de viabilidad se calculó mediante la siguiente ecuación:

$$
\text { Supervivencia celular }(\%)=\frac{A_{570} \text { de las muestras tratadas }}{A_{570} \text { de as muestras no tratadas }} \times 100
$$

\section{RESULTADOS Y DISCUSIÓN}

\section{Solubilidad en agua}

Abreu y Campana-Filho ${ }^{6}$, indican que la solubilidad del polímero O-CMQ está determinada por el equilibrio que implica la protonación de los grupos amino y la disociación de los grupos carboximetilo. Así, las cargas negativas predominarán si el medio es moderadamente alcalino, mientras que las cargas positivas serán predominantes en medio ácido. Aparentemente, el principal factor para determinar la solubilidad del polímero a un $\mathrm{pH}$ dado es el exceso de cargas positivas o negativas, la insolubilidad del polímero es resultado de insuficiente carga. De igual manera, la solubilidad se encuentra estrechamente relacionada 
con el grado de sustitución que presenta el O-CMQ, como lo indica Hjerde ${ }^{10}$, quien reportó que el carboximetilquitosano con un grado de sustitución mayor a $60 \%$ puede disolverse en soluciones acuosas ácidas, neutras y alcalinas.

En la figura 1 se puede observar que el quitosano es totalmente insoluble a los diferentes $\mathrm{pH}$ evaluados. Según Abreu y Campana-Filho ${ }^{6}$, la insolubilidad del quitosano, en medio ácido, se debe a que no habría la suficiente cantidad de ácido en el medio para protonar sus grupos aminos y su insolubilidad en el medio alcalino fue debido a que no presenta carga a $\mathrm{pH}$ mayor a 7,2 .

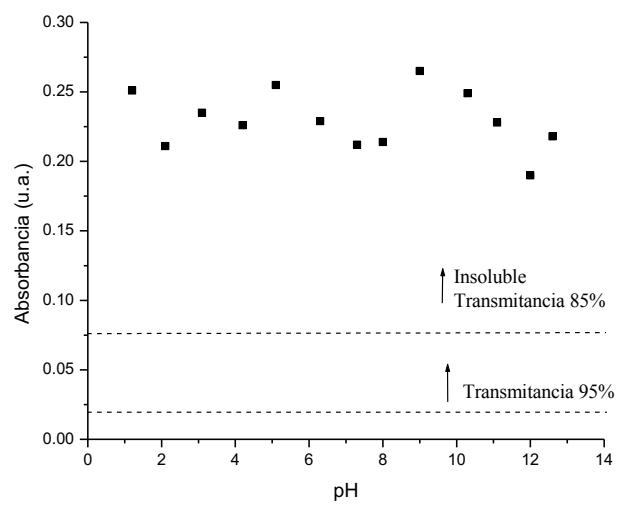

Figura 1. Evaluación de la solubilidad del quitosano (Q) a distintos $\mathrm{pH}$, a $450 \mathrm{~nm}$ de longitud de onda.

En la figura 2, el O-CMQ presenta un rango mayor de solubilidad, excepto a $\mathrm{pH} 5$ y los $\mathrm{pH}$ 4, 6 y 7 pueden ser considerados parcialmente solubles por estar muy cercano al $85 \%$ de transmitancia. Además, se puede observar que existe una buena solubilidad para los $\mathrm{pHs}$ comprendidos entre 1-3 debido a la protonación de los grupos amino y a los pHs 12-13 por la protonación de los grupos carboximetilos.

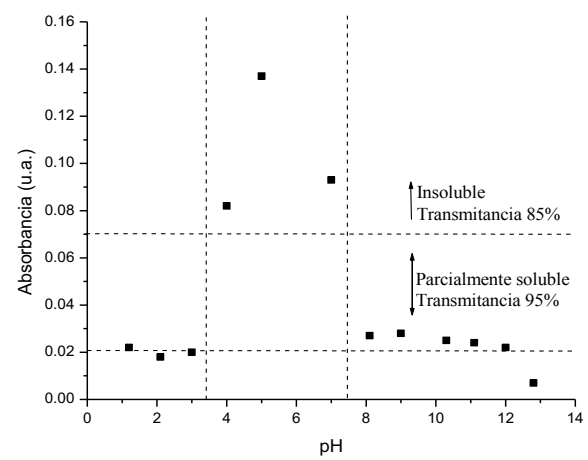

Figura 2. Evaluación de la solubilidad del O-CMQ a distintos pH a $450 \mathrm{~nm}$ de longitud de onda. 
La solubilidad presentada por el O-CMQ se puede atribuir a la presencia de los grupos carboximetilos que contribuyeron a impartir un carácter polielectrolítico aniónico y a la disociación de los grupos amino que le imparten un carácter polielectrolitico catiónico ${ }^{6}$.

\section{Análisis conductimétrico}

La titulación de O-CMQ presenta características complejas debido a la presencia de grupos carboxílico con constantes de acidez similares. La curva de titulación conductimétrica del O-CMQ está curvada en el rango de 9,00 a 19,50 mL de $\mathrm{NaOH}$, a la cual se le ha ajustado cuatro ramas lineales con tres intersecciones, las que llamaremos $V_{1}, V_{2} y_{3}$ (figura 3). El primer punto de intersección $\mathrm{V}_{1}$ (primera rama) corresponde al volumen de base añadido para la neutralización del ácido clorhídrico en exceso, la diferencia de $\mathrm{V}_{2}-\mathrm{V}_{1}$ (segunda rama) corresponde al volumen de la base necesaria para neutralizar los grupos carboximetilo (- $\mathrm{CH}_{2} \mathrm{COOH}$ ) y la diferencia de $\mathrm{V}_{3}-\mathrm{V}_{2}$ (tercera rama) es el volumen de base necesaria para neutralizar los grupos de amonio $\left(-\mathrm{NH}_{3}+\right)$ el cual es proporcional al grado de desacetilación del quitosano original. La cuarta rama lineal de la curva indica el exceso de $\mathrm{NaOH}$. Se utilizaron los valores de los volúmenes equivalentes a la segunda rama $\left(\mathrm{V}_{2}-\mathrm{V}_{1}\right)$ y tercera rama $\left(\mathrm{V}_{3}-\mathrm{V}_{2}\right)$ obtenidos de las curvas de valoración, para calcular el grado de sustitución (GS) utilizando la ecuación 1. El GS obtenido fue 0,92 ( $\mathrm{mol} / \mathrm{mol})$ para el O-CMQ preparado. Campana-Filho ${ }^{11}$ indica que los grados de sustitución altos se deben, fundamentalmente, al exceso de ácido monocloroacético y no a tiempos de reacción prolongados.

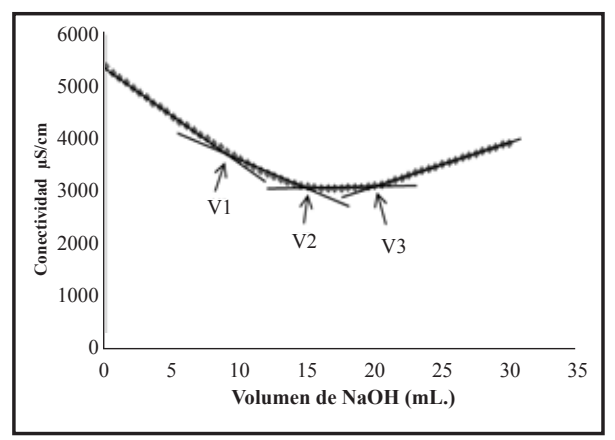

Figura 3. Curva de titulación del O-Carboximetilquitosano.

\section{Masa molecular viscosimétrica (MV)}

La masa molecular viscosimétrica es uno de los parámetros más importantes a tomar en cuenta, ya que con esto se puede determinar la solubilidad y sus posibles aplicaciones.

La viscosidad puede ser descrita en función a la viscosidad intrínseca [ $\eta]$ del polielectrolito y de su concentración, siempre que haya una fuerza iónica suficientemente elevada y no exista interacciones macromoleculares (sistema diluido). En este caso, se puede aplicar la relación de Huggins (ecuación 3) ${ }^{12}$.

$$
\frac{\eta_{s p}}{C}=[\eta]+k_{H} \times[\eta]^{2} \times C
$$


La viscosidad intrínseca del O-CMQ se determinó a partir de la extrapolación de la viscosidad reducida $\left[\eta_{\mathrm{sp}} / \mathrm{C}\right]$ en función a la concentración a dilución infinita, y la masa molar viscosimétrica promedio $\left(\bar{M}_{v}\right)$ se calculó de acuerdo con la ecuación de Mark-Houwink (ecuación 2).

$$
[\eta]=K^{\prime} \times \bar{M}_{v}^{\alpha}
$$

Donde:

“Los valores de los parámetros $\mathrm{K}^{\prime}$ y $\alpha$ utilizados fueron los siguientes: $\mathrm{K}^{\prime}=7,92$ x 10-5, $\alpha=1,00$ " 8 .

En la figura 4 se observa la curva de viscosidad reducida del O-CMQ, en función de la concentración. Los puntos experimentales presentan una correlación lineal elevada $\left(\mathrm{R}^{2}\right.$ $=0,9998$ ), mostrando que las condiciones experimentales empleadas para la determinación de la viscosidad específica fueron adecuadas. Con las ecuaciones 3 y 4 se obtiene la viscosidad intrínseca $[\eta]=221,4 \mathrm{~mL} / \mathrm{g}$ y y la masa viscosimétrica promedio es de $27,95 \mathrm{~g} / \mathrm{mol}$.

El bajo valor de masa viscosimétrica media se debe al comportamiento viscosimétrico de polieletrólitos, tal como el O-CMQ, se espera que en solución los grupos $\mathrm{CH}_{2} \mathrm{COO}-\mathrm{Na}^{+}$ se disocien, generando cargas en las cadenas e interacciones de larga distancia, del tipo electrostáticas. En este caso, los contra-iones se distancian y las cargas localizadas en el macro-ion provocan la expansión de la cadena polimérica, resultando el aumento de la viscosidad $^{13}$.

A pesar de las interacciones entre cadenas, las uniones de hidrógenos y la repulsión electrostática contribuyen a aumentar el peso molecular viscosimétrico medio, pero prevalece la despolimerización de la cadena del polímero causada por el álcali presente en el medio de reacción en la que se preparan las muestras de O-CMC. Por lo tanto, los valores de masa molar media viscosimétrica y viscosidad intrínseca son menores en las muestra de $\mathrm{O}-\mathrm{CMQ}{ }^{13}$.

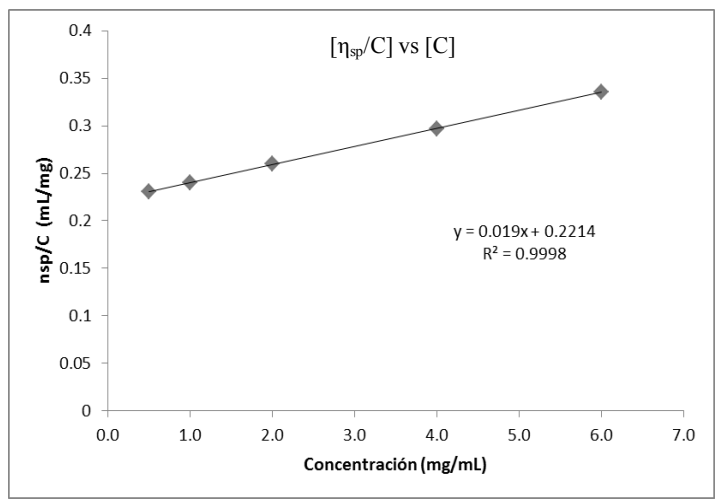

Figura 4. Curva de viscosidad reducida en función de la concentración del O-CMQ en solución de $\mathrm{NaCl} 0,1 \mathrm{~mol} / \mathrm{L}$ a $30^{\circ} \mathrm{C}$ de temperatura. 


\section{Análisis FT/IR del quitosano y O-CMQ}

El espectro FTIR del quitosano (figura 5) muestra bandas de absorción entre 3286,8 y 3356,3 $\mathrm{cm}^{-1}$ correspondientes al estiramiento $\mathrm{O}-\mathrm{H}^{5}$. Por otro lado, estos mismos picos corresponden a un estiramiento N-H característico de una amina secundaria.

En la región comprendida entre 2874,1 a 2920,4 $\mathrm{cm}^{-1}$ se aprecia dos bandas de absorción moderada correspondientes a las vibraciones de tensión $(\mathrm{C}-\mathrm{H})$ propias de grupos $-\mathrm{CH}_{2}-$. Asimismo, en el pico $1647,3 \mathrm{~cm}^{-1}$ muestra una banda de absorción de intensidad moderada correspondiente al estiramiento del $\mathrm{C}=\mathrm{O}$ propio de una amida primaria.

El espectro del O-CMQ (figura 6) muestra: un pico ancho entre 3400 y $3200 \mathrm{~cm}^{-1}$ causado por las vibraciones de los estiramientos O-H y N-H, en la zona de 2895,2 a 2920,4 se aprecia una banda de absorción correspondiente al estiramiento $\mathrm{C}-\mathrm{H}$ propia del grupo $-\mathrm{CH}_{3}$. Asimismo, se muestra un pico fuerte a $1415,8 \mathrm{~cm}^{-1}$ el cual es asignado al estiramiento simétrico de la vibración del grupo -COO-.

En la figura 6 también se observa un pico de absorción a 1585,5 $\mathrm{cm}^{-1}$ correspondiente al estiramiento del $\mathrm{C}=\mathrm{O}$, lo que indica la presencia de un anión carboxílico (-COO-) característico del grupo $-\mathrm{COONa}^{5}$, por el tratamiento con el $\mathrm{NaOH}$ utilizados durante la alcalinización del quitosano. El estiramiento $-\mathrm{C}-\mathrm{O}-$ se encuentra entre 1080 y 1154 . Por lo tanto, el resultado indica la sustitución del grupo hidroxilo del quitosano, verificándose la formación del O-CMQ.

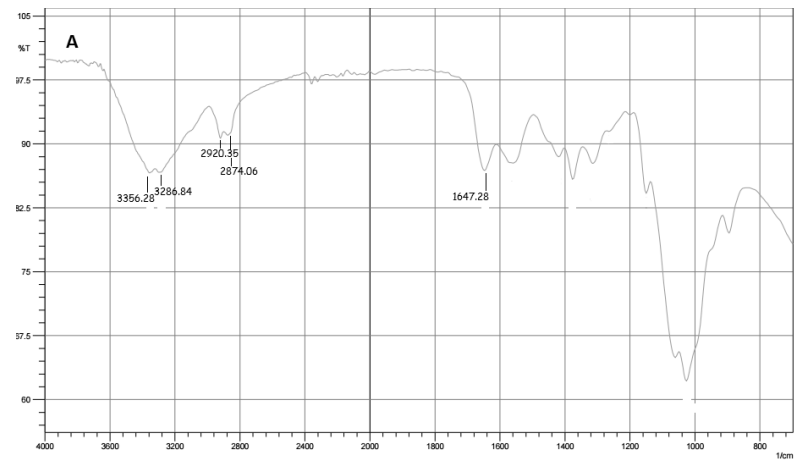

Figura 5. Espectro FTIR de quitosano. 


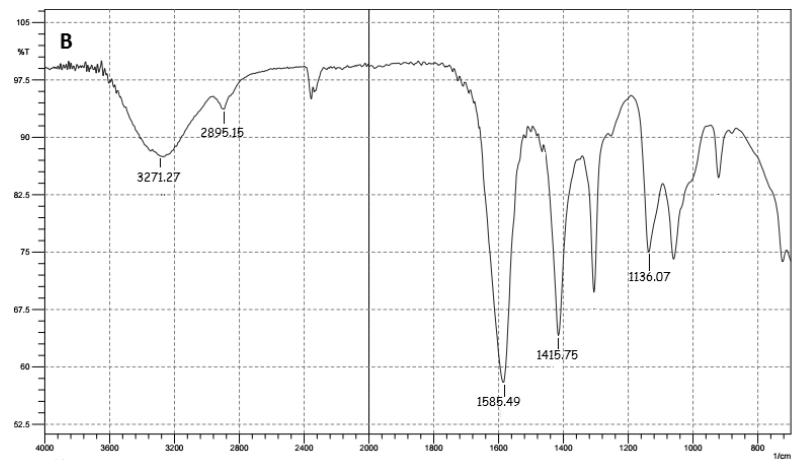

Figura 6. Espectro FTIR del O-CMQ.

\section{Análisis de resonancia magnética nuclear (RMN) del O-CMQ}

En la figura 7 (A), se presentan el espectro de 1H-RMN del O-CMQ. Las señales de protones del O-CMQ aparecieron a 4,49 ppm que corresponde al protón del H3 y a 4,09 ppm correspondiente al protón del H6, cuando está sustituido con el grupo carboximetil.

La figura 7 (B) muestra el espectro de ${ }^{13} \mathrm{C}-\mathrm{RMN}$ del O-CMQ, observándose señales a 70,26 ppm y 69,5 ppm, que son asignadas al grupo $-\mathrm{CH}_{3} \mathrm{COO}$ - sustituidos en los carbonos 6 (C-6) y 3 (C-3), lo que indica alto grado de carboximetilación.

El sustituyente $\mathrm{N}$-carboximetil no se encuentra presente debido a la ausencia de picos a $47 \mathrm{y}$ 168 ppm correspondiente a $\mathrm{N}-\mathrm{CH}_{2}$ y $-\mathrm{COOH}$, respectivamente.

El alto grado de sustitución en el C-6 y en el C-3, se encuentra en concordancia con el resultado del análisis conductimétrico $(0,92 \mathrm{~mol} / \mathrm{mol})$. Chen y Park ${ }^{5}$ reportan que altas sustituciones con grupos carboximetilos provoca un rango de insolubilidad a pHs bajos debido a la aplicación de altas temperaturas durante la síntesis del O-CMQ. 
A

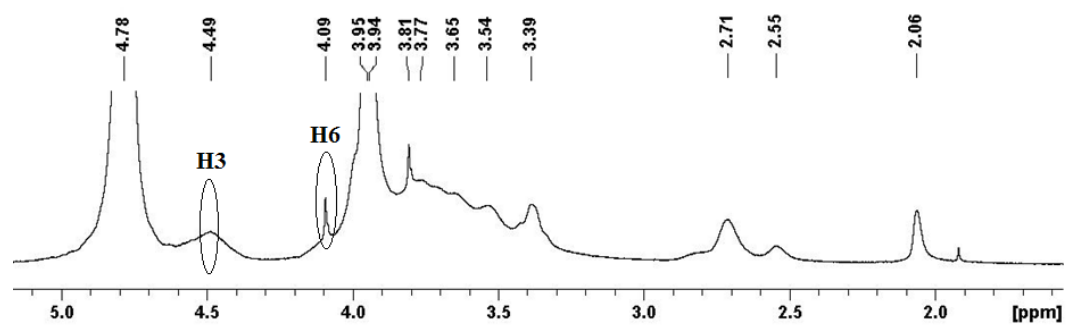

B

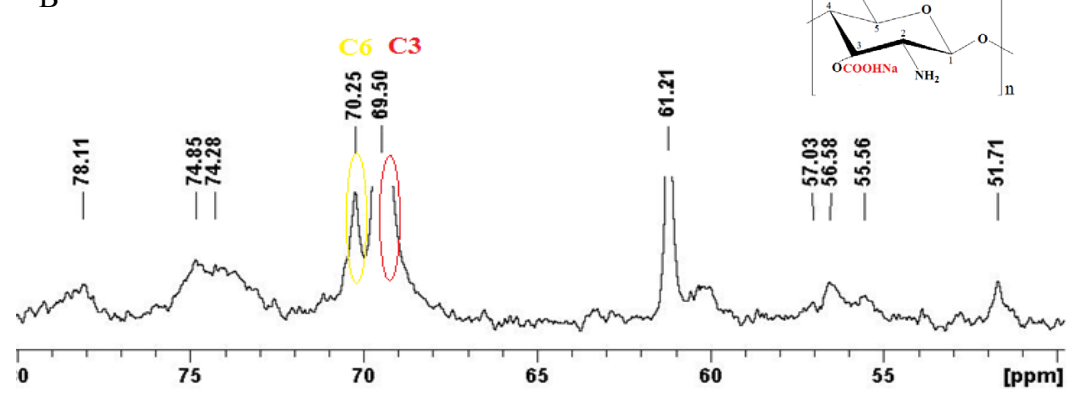

Figura 7 (A). ${ }^{1} \mathrm{H}$ RMN, (B) ${ }^{13} \mathrm{C}$ RMN del O-CMQ

\section{Efecto citoprotector}

El efecto citoprotector se determinó mediante la prueba colorimétrica con el bromuro de 3-(4,5- dimetiltiazol-2-ilo)-2,5-difeniltetrazol (MTT), basada en la capacidad selectiva de la supervivencia celular para reducir el componente tetrazolio de MTT en formazán, que son cristales color púrpura.

Tabla 1. Porcentaje de supervivencia celular del O-CMQ expuestas a concentración de 20uM de $\mathrm{Pb}$

\begin{tabular}{|c|c|}
\hline Muestras & $\begin{array}{c}\text { \% Supervivencia } \\
\text { celular }\end{array}$ \\
\hline Blanco & 100,00 \\
\hline O-CMQ $0,5 \mathrm{mg} / \mathrm{mL}$ & 71,53 \\
\hline $\mathrm{Pb} 41,44 \mathrm{mg} / \mathrm{mL}$ & 55,56 \\
\hline $\mathrm{Pb} 41,44 \mathrm{mg} / \mathrm{mL} / \mathrm{CMQ} 0,5 \mathrm{mg} / \mathrm{mL}$ & 63,89 \\
\hline $\mathrm{Pb} 82,88 \mathrm{mg} / \mathrm{mL}$ & 53,47 \\
\hline $\mathrm{Pb} 82,88 \mathrm{mg} / \mathrm{mL} / \mathrm{CMQ} 0,5 \mathrm{mg} / \mathrm{mL}$ & 53,82 \\
\hline
\end{tabular}


En la tabla 1 se muestra los resultados de la supervivencia celular, expuestos a concentraciones de 41,44 y 82,88 mg/mL de plomo. El O-CMQ atenúa la toxicidad del $\mathrm{Pb}$ en un $8,33 \%$ a una concentración de 41,44mg/Ml, en cambio para una concentración de plomo de $82,88 \mathrm{mg} /$ $\mathrm{mL}$ no hay protección. El efecto de atenuación que presenta el O-CMQ estaría relacionado directamente con el grado de sustitución del O-CMQ, mediante un mecanismo de quelación a través de átomos de oxígeno y nitrógeno coordinados con los iones $\mathrm{Pb}$ (II) en la cadena del polímero del O-CMQ ${ }^{3}$.

\section{CONCLUSIONES}

Se preparó O-Carboximetilquitosano (O-CMQ) por reacción de quitosano pre-alcalinizado con ácido monocloroacético, las condiciones de reacción fueron las siguientes: tiempo de alcalinización, 8h; proporción ácido monocloroacético y quitosano, 6:1; temperatura de reacción, $50^{\circ} \mathrm{C}$; tiempo de reacción, $6 \mathrm{~h}$ y agitación constante de $2000 \mathrm{rpm}$. El O-CMQ obtenido fue soluble en un amplio rango de $\mathrm{pH}$ comprendido entre 1-13, con excepción pH 5. El grado de sustitución del O-CMQ fue de $0,92 \mathrm{~mol} / \mathrm{mol}$ del total de monómeros.

El FT-IR, confirmó la formación del O-CMQ, por la presencia de un pico de absorción a $1585,5 \mathrm{~cm}^{-1}$ correspondiente al estiramiento del $\mathrm{C}=\mathrm{O}$, lo que indica la presencia de un anión carboxílico (-COO-). Por RMN de $1 \mathrm{H}$ y 13C se confirmó la formación del O-CMQ, observándose que la sustitución de los grupos carboximetilos ocurrió en los hidroxilos que están presentes en el carbono 3 y 6 . Se demostró que el O-CMQ, a una concentración de $50 \mathrm{mg} / \mathrm{mL}$, incrementó la supervivencia de las células en un $8,33 \%$ frente a la toxicidad del $\mathrm{Pb}$ (II) a una concentración de $41 \mathrm{mg} / \mathrm{l}$.

\section{AGRADECIMIENTO}

Se agradece al Programa Nacional de Innovación para la Competitividad y Productividad, Innóvate Perú, por los fondos RNR asignados al proyecto: "Funcionalización de textiles de algodón con nanocompositos NPsAg-carboximetilquitosano para mejorar las propiedades antibacterianas y antifúngicas con potencial aplicación en la fabricación de prendas de uso hospitalario" CONVENIO 396-PNICP-PIAP-2014.

De igual manera se agradece a la Universidad Nacional de San Agustín de Arequipa porque permitió el desarrollo de la investigación en el Laboratorio del Departamento de Química. 


\section{REFERENCIAS BIBLIOGRÁFICAS}

1. Rinaudo M. Chitin and chitosan: Properties and applications. Prog Polym Sci. 2006; 31:603-632.

2. Cravotto G, Tagliapietra S, Robaldo B, Trotta M. Chemical modification of chitosan under high-intensity ultrasound. Ultrason Sonochem. 2005; 12:95-98.

3. Sun S, Wang L, Wang A. Adsorption properties of crosslinked carboxymethyl-chitosan resin with $\mathrm{Pb}(\mathrm{II})$ as template ions. J Hazard Mat. 2006; 136(3): 930-937.

4. Mourya VK, Inamdar NN, Tiwari Ashutosh. Carboxymethyl chitosan and its applications. Adv Mater Lett. 2010; 1(1):11-33.

5. Chen X-G, Park H-J. Chemical characteristic of O-carboxymethyl chitosans related to the preparation condition. Carbohydr Polym. 2003; 53:355-359.

6. Abreu FR, Campana-Filho SP. Characteristics and properties of carboxymethylchitosan. Carbohydr Polym. 2009; 75:214-221.

7. Vaghani SS, Patel MM, Satish CS. Synthesis and characterization of $\mathrm{pH}$-sensitive hydrogel composed of carboxymethyl chitosan for colon targeted delivery of ornidazole. Carbohydr Res. 2012; 347:76-82.

8. Ge H-C, Luo D-K. Preparation of carboxymethyl chitosan in aqueous solution under microwave irradiation. Carbohydr Res. 2005; 340:1351-1356.

9. Anitha A, Divya RVV, Krishna R, Sreeja V, Selvamurugan N, Nair SV, et al. Synthesis, characterization, cytotoxicity and antibacterial studies of chitosan, O-Carboxymethyl and N,O-carboxymethyl chitosan nanoparticles. Carbohydr Polym. 2009; 78:672-677.

10. Hjerde RJN, Vårum KM, Grasdalen H, Tokura S, SmidsrØd O. Chemical composition of O-(carboxymethyl)- chitins in relation to lysozyme degradation rates. Carbohydr Polym. 1997; 34:131-139.

11. Campana-FilhoSP,AbreuFR.PreparationandcharacterizationofCarboxymethylchitosan. Polímeros: Ciência e Tecnología. 2005; 5(2):79-83.

12. Roberts GAF. Chitin chemistry. London: Mc Millan; 1992. p. 350.

13. De Souza e Silva Daniella. Estudos físico-químicos de O-carboximetilação de quitosana [tesis]. São Carlos: Universidade de São Paulo; 2011. 